\title{
The epidemics of obesity and type 2 diabetes: Implications for noninvasive cardiovascular imaging
}

\author{
George A. Beller, MD
}

\begin{abstract}
Although the age-adjusted mortality rate for coronary heart disease (CHD) has fallen by $60 \%$ since the 1960s, the prevalence of CHD is increasing. This is a result of the increase in the elderly population and the epidemics of obesity and type 2 diabetes. Presently, 97 million adults in the United States are overweight (body mass index of 25 to 29.9) and 44.3 million are obese. Obesity in the United States increased by a staggering $5.6 \%$ just from 2000 to 2001 and increased by $74 \%$ since 1991. Health care costs are $37 \%$ higher for overweight and obese individuals, and such costs add $\$ 732$ to the annual medical bills for every American. What is alarming is the fact that worldwide approximately 22 million children under the age of 5 years are overweight. In 2000, 22\% of the school children in the United States were overweight, and the number of overweight children has tripled between 1970 and 2000. It has now reached $15 \%$ of those aged 6 to 19 years. In addition, 910,000 teenagers in the United States fulfill the criteria for metabolic syndrome $(6.1 \%$ are male and $2.1 \%$ are female). ${ }^{1}$
\end{abstract}

One of the major underlying causes of the obesity epidemic is physical inactivity in our population. Fifty percent of youths aged 12 to 21 years are not vigorously active on a regular basis, and twenty-five percent of Americans are sedentary. It is estimated that only $15 \%$ of US adults engage in regular physical activity, defined as exercising 3 times a week for at least 20 minutes.

Consequent to the epidemic of obesity and other factors, approximately 17 million Americans have diabetes, and many others have undiagnosed diabetes. Approximately $75 \%$ to $80 \%$ of these diabetic persons will die of cardiovascular disease. According to the Communicable Disease Center, 45 to 50 million US residents could have diabetes by 2050 . Essentially, 1 in 3 children born in 2000 will become diabetic unless many more people eat less and exercise more. The average American now consumes 530 calories more per day than was consumed in 1970, and approximately 600 million Big Macs and 20 billion hot dogs are consumed each year. ${ }^{2}$ Patients with diabetes and no CHD have the same

J Nucl Cardiol 2004;11:105-6.

Copyright $\odot 2004$ by the American Society of Nuclear Cardiology. $1071-3581 / 2004 / \$ 30.00+0$

doi:10.1016/j.nuclcard.2004.01.006 risk for future cardiac death as nondiabetic persons with prior myocardial infarction. Diabetes increases the risk of cardiac events and death in patients with documented CHD. Diabetic persons have higher mortality and morbidity rates than nondiabetic persons after acute coronary syndromes and have a worse prognosis after percutaneous coronary intervention and coronary bypass surgery.

From these demographic data and prevalence statistics, it can be expected that many diabetic persons presenting with symptoms suggestive of coronary artery disease (CAD) will be referred for diagnostic noninvasive testing. Coincident with determining whether significant CAD is present will be the process of risk stratification to identify those who deserve further invasive evaluation with a view toward revascularization. Stress myocardial perfusion imaging has proven valuable in both diagnostic and prognostic evaluations of diabetic patients. Diabetic patients with mildly or moderately to severely abnormal scans have a significantly higher cardiac event rate compared with nondiabetic persons with a similar extent and severity of perfusion abnormalities. ${ }^{3}$ Myocardial perfusion imaging variables provide major incremental value for risk stratification in diabetic persons. ${ }^{4}$ In this study diabetic women with ischemia in two or more coronary vascular territories had only a $60 \%$ 3 -year survival free of cardiac death or nonfatal infarction. Diabetic men had a 79\% event-free survival rate. Diabetic persons with normal perfusion scans have a higher event rate than nondiabetic persons with normal scans. Adenosine myocardial perfusion imaging has also demonstrated incremental prognostic value for predicting cardiac death in both diabetic men and women. ${ }^{5}$ Thus our nuclear cardiology laboratories can expect to see a greater number of symptomatic diabetic persons who will be referred for noninvasive determination of the presence and extent of CAD. On the basis of the increasing incidence of the metabolic syndrome and type 2 diabetes worldwide and its consequent increase in CHD in the population, our noninvasive imaging laboratories should experience an increasing volume of patients.

A major area of current interest is the role of noninvasive imaging in asymptomatic type 2 diabetic persons, particularly those who have at least one or two additional major CAD risk factors. Recently, the results of the Detection of Ischemia in Asymptomatic Diabetics 
(DIAD) study were reported by the study investigators. The major objective of this study was to assess the prevalence and clinical predictors of silent myocardial ischemia in asymptomatic type 2 diabetic persons. Currently, the American Diabetes Association's screening guidelines suggest that standard treadmill testing be undertaken in diabetic persons with two or more CAD risk factors. ${ }^{6}$ In the DIAD study, approximately 500 patients were randomized to adenosine technetium $99 \mathrm{~m}$ sestamibi single photon emission computed tomography (SPECT) testing for identifying silent ischemia. Surprisingly, 1 out of every 5 of these asymptomatic patients with type 2 diabetes had silent ischemia. The investigators reported that the traditional risk factors were not predictive of adenosine SPECT abnormalities, nor was the presence of inflammatory markers. The only variable that seemed to be associated with abnormal SPECT studies was cardiac autonomic dysfunction.

Other noninvasive screening tests are also being applied to the asymptomatic diabetic population, including coronary artery calcification imaging with electron beam computed tomography or multislice computed tomography scanning, measurement of intimal-medial thickness by carotid B-mode ultrasound, and cardiac magnetic resonance imaging. The challenge to investigators in this area is to institute prospective clinical studies such as the DIAD study to better determine which subpopulation of the large and expanding asymptomatic diabetic population will benefit in a cost-effective manner from noninvasive testing. We look forward to follow-up data from the DIAD study, as one half of the patients did not undergo cardiac testing, and the subsequent comparison of outcomes from those who did and did not undergo adenosine SPECT could assist in evaluating the potential benefit of noninvasive imaging in this asymptomatic population. The issue of screening intermediate-risk asymptomatic subjects for the presence of coronary atherosclerosis or significant silent ischemia is a controversial one, as it is estimated that $40 \%$ of the
US population has either one major risk factor or a positive family history of $\mathrm{CAD}^{7}$ Good research in the area of the cost-effectiveness of screening with expensive technology is surely warranted in the asymptomatic diabetic population, in whom a high prevalence of premature coronary atherosclerosis has been documented and where 1 of 5 patients exhibit silent ischemia on stress perfusion imaging. One major prerequisite of such screening approaches is that early detection should result in a curative potential at early stages of the disease, improved patient outcomes, and a favorable tradeoff between risks, benefits, and costs. ${ }^{8}$

\section{References}

1. Cook S, Weitzman M, Auinger P, Nguyen M, Dietz WH. Prevalence of a metabolic syndrome phenotype in adolescents. Findings from the Third National Health and Nutrition Examination Survey, 1988-1994. Arch Pediatr Adolesc Med 2003;157:821-7.

2. Gorman C, Bjerklie D, Bower A, Locke L, Sieger M, Sikora F, Booth Thomas C. How to eat smarter. Time magazine 2003;160: 48-59.

3. Kang X, Berman DS, Lewin HC, et al. Incremental prognostic value of myocardial perfusion single photon emission computed tomography in patients with diabetes mellitus. Am Heart J 1999;138:102532.

4. Giri S, Shaw LJ, Murthy DR, et al. Impact of diabetes on the risk stratification using stress single-photon emission computed tomography myocardial perfusion imaging in patients with symptoms suggestive of coronary artery disease. Circulation 2002;105:32-40.

5. Berman DS, Kang X, Hayes SW, et al. Adenosine myocardial perfusion single-photon emission computed tomography in women compared with men. Impact of diabetes mellitus on incremental prognostic value and effect on patient management. J Am Coll Cardiol 2003;41:1125-33.

6. American Diabetes Association. Consensus development conference on the diagnosis of coronary heart disease in people with diabetes: 10-11 February 1998, Miami, Florida. Diabetes Care 1998;21:1551-9.

7. Greenland P, Gaziano JM. Selecting asymptomatic patients for coronary computed tomography or electrocardiographic exercise testing. N Engl J Med 2003;349:465-73.

8. Hunink MGM, Gazelle GS. CT screening: a trade-off of risks, benefits, and costs. J Clin Invest 2003;111:1612-9. 